\title{
A New Analysis Technique of Hybrid Production Lines
}

\author{
Jun Liu, Qinying Fan, and Junping Kong
}

\begin{abstract}
A new system aggregation technique was proposed to analyze hybrid production lines. Different from the traditional techniques, new lines consisting of "equivalent" machines were set up and the parameters of machines were renewed repeatedly rather than fixed in the process of whole aggregation. Meanwhile, forward aggregation and backward aggregation alternately proceeded until steady system parameters of production lines were gotten. The comparison analysis between the technique and the traditional aggregation technique was done by numerical experiments. The advantages and applying circumstance of the new aggregation technique were also specified by numerical results.
\end{abstract}

Index Terms-Hybrid production line, System performance, Aggregation technique.

\section{INTRODUCTION}

It is well known that a production system is generally a hybrid system [1]. An amount of work has been devoted to the modeling and analysis of transfer and production lines using analytical methods since the early 1950 's because of their economic importance as well as academic interest. A comprehensive survey presented by Dallery and Gershwin [2] provides extensive and elaborate reviews up to 1992 . Li J. et al. [3] offers a supplementary review up to 2007. Readers can also refer to some books [4] on how to model and analyze transfer lines. The two-machine lines are the basis of researching longer production lines. For the models of two machines, such as discrete model, synchronous model, asynchronous model, continuous model, homogeneous model, non-homogeneous model, etc, the approximate solutions have been obtained by some scholars [5]. Now further works have been devoted to the analysis of longer production lines [6]. However, it is very difficult (is not hopeless) to obtain exact analytical solutions of production lines with more than three machines. The major reason is that the system states increase exponentially with the increase of machines. The curse of dimensionality makes such problems intractable even if more powerful computers are available. It appears that "it is difficult to program, ill-behaved, and not extendable to larger problems" [7]. So far to value and analyze the longer production lines, three main approximate techniques have been proposed: decomposition method [7], aggregation method [8] and simulation method.

The idea of decomposition method is to decompose the

Manuscript received March 22, 2014; revised May 29, 2014. This work was supported by National Natural Science Fund (No.51265032) and Program for Changjiang Scholars and Innovative Research Team in University (No.IRT1140).

Jun Liu is with Lanzhou University of Technology, 730050, Lanzhou, China (e-mail: lzhjliu@126.com).

Qinying Fan and Junping Kong are with Lanzhou University of Technology, 730050, Lanzhou, China (e-mail: 958174577@qq.com). original production line into a set of two-machine lines, and the behavior of the two-machine lines closely approximates that of the original production line. Many scholars do a great deal of work to increase the efficiency of the technique, such as Dallery Y. and Bihan H. L. [9], Colledani M. and Tolio T. [10], etc. The technique seem to find a balance between complexity and reliability, for example, more simplified and high convergent algorithms such as ADDX,BDDX, etc, are offered and developed [11], [12]. Moreover, the method has been developed and widely utilized to study production lines with complex construction, e.g. parallel lines [13], assembly/disassembly lines [14], closed loop system [15], etc.

The idea of aggregation method is to replace a buffer and the two surrounding machines by a single "equivalent" machine, and step by step, the whole line can be aggregated into a single "equivalent" machine so that the system performance can be estimated by the last "equivalent" machine. Compared with decomposition method, the aggregation method has larger analysis error on some models of production line. But it can be utilized directly to analyze both homogeneous production line and non-homogeneous production line without any transformation of model. In this respect, the decomposition method cannot do it. The method is widely utilized by following scholars. The simulation method is comparatively accurate, whereas it is time-consuming.

Motivated by the literature [16], the paper proposes a new aggregation technique. Different from traditional aggregation method, the parameters of first machine and last machine are renewed by "equivalent" machine gotten in aggregation process. A new line consisting of "equivalent" machines is set up after a forward (or backward) aggregation process. Forward aggregation and backward aggregation alternately proceed until steady system parameters of production lines are gotten. For the convenience of comparing the technique with traditional aggregation method, the data in the literature [6] are utilized in numerical experiments. The new aggregation technique proposed in the paper is specified by numerical results. The new aggregation technique complements and develops the system analysis techniques of hybrid production lines.

\section{Problem Statements}

A typical production line which consists of $N$ machines $M_{i}, i \in\{1,2,3, \cdots, N\}$ and $N-1$ buffers $B_{i}, i \in\{1,2,3, \cdots, N-1\}$ is shown in Fig.1. New material or parts flow from outside of the system to the first machine $M_{1}$, then to the buffer $B_{i}, i \in\{1,2, \cdots, N-1\}$, machine $M_{i}, i \in\{2,3, \cdots, N\}$, finally out of the system. 


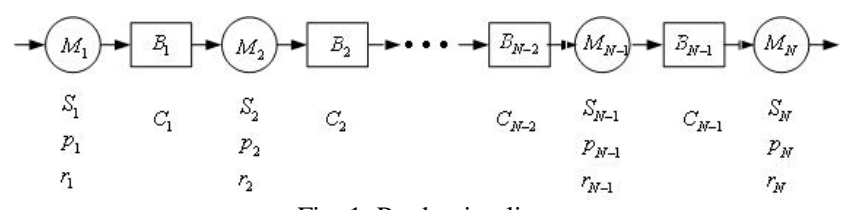

Fig. 1. Production line

The machines are all unreliable. Assume that the machine $M_{i}, i \in\{1,2,3, \mathrm{~L}, N\}$ up and down times is independent and exponentially distributed with means $1 / p_{i}$ and $1 / r_{i} \quad i \in\{1,2, \mathrm{~L}, N\} \quad$ respectively. $C_{i}, i \in\{1,2, \mathrm{~L}, N-1\}$ is the buffer size of buffe $B_{i}, i \in\{1,2, \mathrm{~L}, N-1\}$. Blockage occurs if the machine $M_{i}, i \in\{1,2,3, \mathrm{~L}, N-1\}$ is operational and the buffer level $n_{i}, i \in\{1,2, \mathrm{~L}, N-1\}$ of the next downstream buffer $B_{i}, i \in\{1,2, \mathrm{~L}, N-1\}$ reaches its capacity $C_{i}, i \in\{1,2, \mathrm{~L}, N-1\}$. "Starvation" occurs if the machine $M_{i}, i \in\{2,3, \mathrm{~L}, N\}$ is operational and the buffer level $n_{i}, i \in\{1,2, \mathrm{~L}, N-1\}$ of the adjacent upstream buffer $B_{i}, i \in\{1,2, \mathrm{~L}, N-1\}$ is zero. Let $S_{i}, i \in\{1,2,3, \mathrm{~L}, N\}$ denote the maximum production rate of the machine $M_{i}, i \in\{1,2,3, \mathrm{~L}, N\}$. Let $\alpha_{i}(t) \in\{0,1\}, i \in\{1,2, \mathrm{~L}, N\}$ denote the state of the machine $M_{i}, i \in\{1,2,3, \mathrm{~L}, N\}$ and be a continuous-time Markov process respectively. The equation $\alpha_{i}(t)=1, i \in\{1,2, \mathrm{~L}, N\}$ indicates that the machine $M_{i}, i \in\{1,2,3, \mathrm{~L}, N\}$ is operational and $\alpha_{i}(t)=0, i \in\{1,2, \mathrm{~L}, N\}$ indicates the machine is under repair.

Assume that there are always parts available at the input of the system and spaces available at the output of the system. Meanwhile, assume that the failures are all operation dependent. Then $e_{i}=S_{i} r_{i} /\left(r_{i}+p_{i}\right), i \in\{1,2,\llcorner\cdots, N\}$

is the average independent production rate of the machine $M_{i}, i \in\{1,2,3, \mathrm{~L}, N\}$. The average production rate of the machine $M_{i}, i \in\{1,2,3,\llcorner\cdots, N\}$ in the line is

$E_{i}=S_{i} r_{i} /\left(r_{i}+p_{i}\right) \operatorname{prob}\left(\alpha_{i}=1, n_{i-1}>0, n_{i}<C_{i}\right), i \in\{1,2,3, \cdots, N\}$.

Due to the line is a conserve system, the system throughput $E$ of the line in a long run is as follows:

$$
E=E_{1}=E_{2}=\cdots=E_{N}
$$

The system average buffer level $\overline{n_{i}}$ of the buffer $B_{i}, i \in\{1,2,3, \cdots, N-1\}$ is as follows:

$$
\overline{n_{i}}=\sum_{n=0}^{C_{i}} n_{i} \sum_{\alpha_{i}, \alpha_{i+1}=1,0} p\left(n_{i}, \alpha_{i}, \alpha_{i+1}\right), i \in\{1,2, \ldots, N-1)
$$

The production system throughput $E$ and average buffer level $\overrightarrow{n_{i}}, i \in\{1,2,3, \cdots, N-1\}$ are main system performance parameters of production lines. Our objective in the paper is to estimate the system throughput of lines by a new aggregation method.

\section{A New System AgGREGation Method}

\section{A. Traditional System Aggregation Method}

For analysis of production line with many unreliable machines, the literature [8] has proposed an aggregation method.

The idea of the method is to replace a buffer and the two surrounding machines by a single "equivalent" machine whose behavior is similar with the original two-machine line. Step by step, for a N-machine line, an system aggregated model consisting of just one "equivalent" machine can be obtained finally. Then the throughput of the $\mathrm{N}$-machine system is gotten by the average production rate of the final "equivalent" machine. The aggregation process from upside of the line is as shown in Fig. 2.

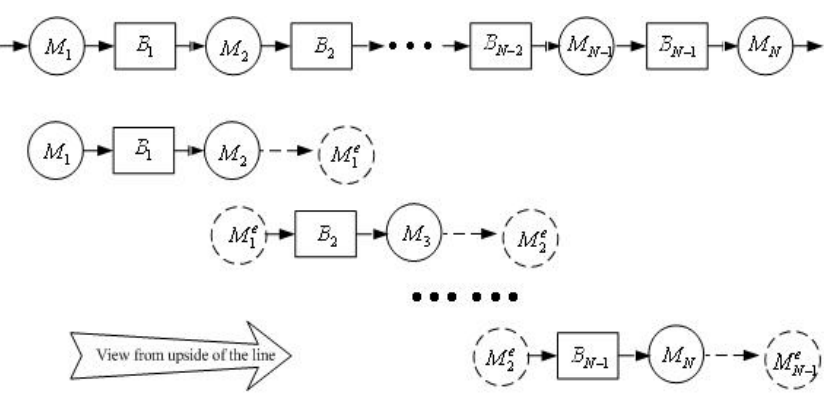

Fig. 2. Aggregation process.

In Fig. 2, $M_{i}^{e}, i=1,2, . . N-1$ is the corresponding "equivalent" machine. The method view from upstream is called forward aggregation. The method view from downstream is called backward aggregation. If the hybrid method is utilized, then it is called "hybrid aggregation". For the advantages, disadvantages or more details of the methods, please refers to [8], [17], etc, for more help.

\section{B. A New System Aggregation Method}

The aggregation process of traditional aggregation method stops when the whole production line is aggregated into an "equivalent" machine and the throughput of the system can be obtained by the parameters of the "equivalent" machine.
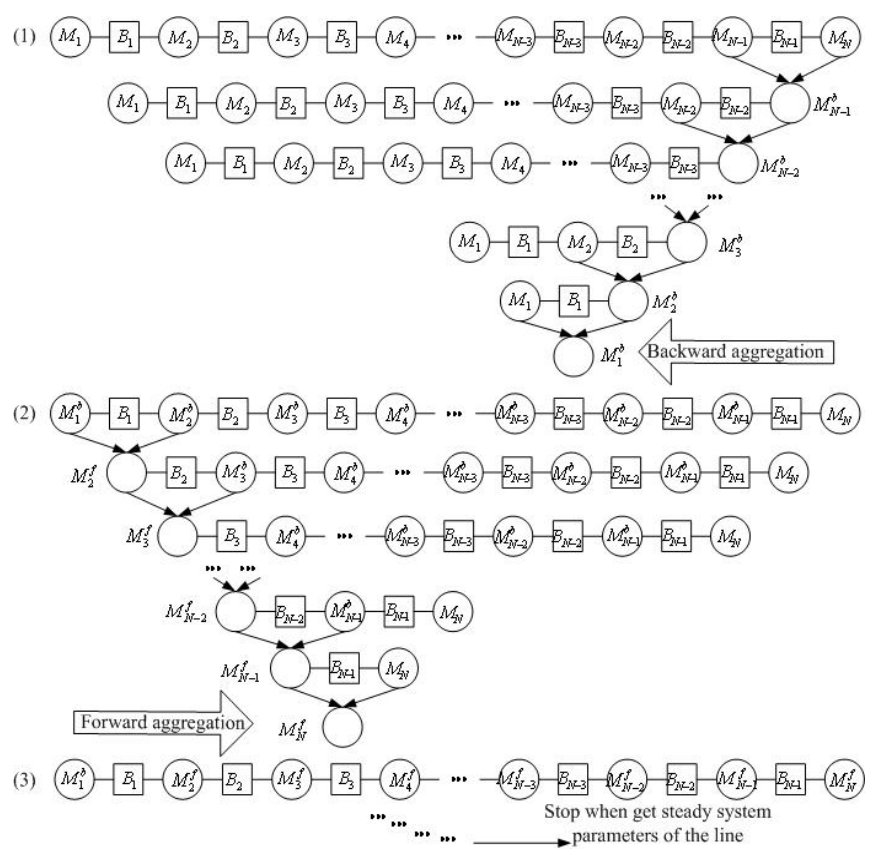

Fig. 3. New aggregation technique. 
Based on the principle of system aggregation, a new system aggregation method motivated by the literature [16] is proposed here. Fig. 3 shows the new aggregation technique started from the backward aggregation.

Firstly, the backward aggregation is adopted. The pseudo "equivalent" machine $M_{N-1}^{b}$ in Fig. 3 is obtained by the aggregation of machine $M_{N}, M_{N-1}$ and the buffer $B_{N-1}$ (the superscript $b$ of $M_{N-1}^{b}$ means that the backward aggregation is going) $M_{N-2}^{b}$ is obtained by the machine $M_{N-1}^{b}, M_{N-2}$ and the buffer $B_{N-2}$. And so on, until the whole line is aggregated into a pseudo "equivalent" machine $M_{1}^{b}$.

Secondly, a new line is set up by the "equivalent" machines $M_{1}^{b}, M_{2}^{b}, \cdots, M_{N-1}^{b}$ obtained in the above backward aggregation process and the last machine $M_{N}$ of the original line. Then the forward aggregation subsequently is adopted to analyze the new pseudo-line. The "equivalent" machine $M_{2}^{f}$ in Fig. 3 is obtained by the aggregation of machine $M_{1}^{b}, M_{2}^{b}$ and the buffer $B_{1}$ (the superscript $f$ of $M_{2}^{f}$ means that the forward aggregation is going).$M_{3}^{f}$ is obtained by the aggregation of machine $M_{2}^{f}, M_{3}^{b}$ and the buffer $B_{2}$. And so on, until the whole pseudo-line is aggregated into an "equivalent" machine $M_{N}^{f}$.

Thirdly, another new pseudo-line is set up by utilizing the "equivalent" machines $M_{2}^{f}, M_{3}^{f}, \cdots, M_{N}^{f}$ obtained in the second step and the machine $M_{1}^{b}$. Then the processing similar to the above processes goes on until steady system parameters of production lines are obtained.

The new aggregation technique is different from the traditional method. In the literature [8], the aggregation process of traditional method is one-way aggregation. However, the forward aggregation and backward aggregation alternately proceeded in the new method. Furthermore, our method builds new lines by pseudo "equivalent" machines obtained in the process of aggregation, meanwhile, the parameters of all machines including the first and the last machine in the line are not fixed and renewed repeatedly by aggregation.

When upstream machine $M_{i}$, downstream machine $M_{i+1}$ and buffer $B_{i}$ are replaced by an "equivalent" machine $M_{i}^{e}$ in aggregation method, the following parameters of $M_{i}^{e}$ can be deduced by combining the results of the literature [8] with the results of the literature [16]:

1) If $S_{i+1} \geq S_{i}$

a) When $S_{i+1}=S_{i}$

$\rho_{i}^{e}=\left\{\begin{array}{ll}\frac{r_{i} r_{i+1} S_{i}\left[p_{i}\left(p_{i+1}+r_{i+1}\right)-p_{i+1}\left(p_{i}+r_{i}\right) e^{-\beta C_{i}}\right]}{\left(p_{i}+r_{i}\right)\left(p_{i+1}+r_{i+1}\right)\left(p_{i} r_{i+1}-p_{i+1} r_{i} e^{-\beta C_{i}}\right)} & \text { if } \frac{p_{i}}{r_{i}} \neq \frac{p_{i+1}}{r_{i+1}} \\ \frac{S_{i} r_{i+1}^{2}\left(r_{i}+r_{i+1}\right)+C_{i} r_{i} r_{i+1}\left(p_{i+1}+r_{i+1}\right)^{2}}{\left(p_{i+1}+r_{i+1}\right)^{2}\left[S_{i}\left(r_{i}+r_{i+1}\right)+C_{i} r_{i}\left(p_{i+1}+r_{i+1}\right)\right]} S_{i} & \text { if } \frac{p_{i}}{r_{i}}=\frac{p_{i+1}}{r_{i+1}}\end{array}\right.$,

$p_{i}^{e}=\frac{r_{i} \rho_{i}^{e}\left(S_{i}-\rho_{i}^{e}\right)^{2}\left(r_{i}+p_{i}\right)^{2}\left(r_{i+1}+p_{i+1}\right)}{S_{i}^{2} p_{i} r_{i} \rho_{i}^{e}\left(r_{i+1}+p_{i+1}\right)+S_{i+1}^{2} r_{i+1} p_{i+1}\left(r_{i}+p_{i}\right)^{2}\left[S_{i} r_{i}-\rho_{i}^{e}\left(r_{i}+p_{i}\right)\right]}$,

$$
r_{i}^{e}=\frac{r_{i}\left[\rho_{i}^{e}\right]^{2}\left(S_{i}-\rho_{i}^{e}\right)\left(r_{i}+p_{i}\right)^{2}\left(r_{i+1}+p_{i+1}\right)}{S_{i}^{2} p_{i} r_{i} \rho_{i}^{e}\left(r_{i+1}+p_{i+1}\right)+S_{i+1}^{2} r_{i+1} p_{i+1}\left(r_{i}+p_{i}\right)^{2}\left[S_{i} r_{i}-\rho_{i}^{e}\left(r_{i}+p_{i}\right)\right]} .
$$

where $\rho_{i}^{e}, p_{i}^{e}$ and $r_{i}^{e}$ is the production rate, breakdown rate and repair rate of machine $M_{i}^{e}$ respectively.

$\beta=\frac{\left(p_{i}+r_{i}+p_{i+1}+r_{i+1}\right)\left(p_{i} r_{i+1}-p_{i+1} r_{i}\right)}{\left(r_{i}+r_{i+1}\right)\left(p_{i}+p_{i+1}\right) S_{i}}$.

b) When $S_{i+1}>S_{i}$

$\rho_{i}^{e}=\frac{S_{i+1} e_{i+1} A e^{\gamma_{1} C_{i}}+S_{i} e_{i} B e^{\gamma_{2} C_{i}}+S_{i} e_{i} D e^{-\gamma_{2} C_{i}}}{A e^{\gamma_{1} C_{i}}+B e^{\gamma_{2} C_{i}}+D e^{-\gamma_{2} C_{i}}}$,

$p_{i}^{e}=\frac{r_{i} \rho_{i}^{e}\left(S_{i}-\rho_{i}^{e}\right)^{2}\left(r_{i}+p_{i}\right)^{2}\left(r_{i+1}+p_{i+1}\right)}{S_{i}^{2} p_{i} r_{i} \rho_{i}^{e}\left(r_{i+1}+p_{i+1}\right)+S_{i+1}^{2} r_{i+1} p_{i+1}\left(r_{i}+p_{i}\right)^{2}\left[S_{i} r_{i}-\rho_{i}^{e}\left(r_{i}+p_{i}\right)\right]}$,

$r_{i}^{e}=\frac{r_{i}\left[\rho_{i}^{e}\right]^{2}\left(S_{i}-\rho_{i}^{e}\right)\left(r_{i}+p_{i}\right)^{2}\left(r_{i+1}+p_{i+1}\right)}{S_{i}^{2} p_{i} r_{i} \rho_{i}^{e}\left(r_{i+1}+p_{i+1}\right)+S_{i+1}^{2} r_{i+1} p_{i+1}\left(r_{i}+p_{i}\right)^{2}\left[S_{i} r_{i}-\rho_{i}^{e}\left(r_{i}+p_{i}\right)\right]}$.

where

$e_{i}^{\prime}=\frac{r_{i}}{r_{i}+p_{i}}, \quad e_{i+1}^{\prime}=\frac{r_{i+1}}{r_{i+1}+p_{i+1}}, A=r_{i} Q^{2}+r_{i} Q\left[S_{i}\left(r_{i}+r_{i+1}+p_{i+1}\right)-S_{i+1}\left(r_{i}+r_{i+1}+p_{i}\right)\right]$,

$B=r_{i+1} p_{i} S_{i+1}\left[\left(S_{i+1}-S_{i}\right)\left(r_{i}-r_{i+1}\right)-\left(S_{i+1} p_{i}+S_{i} p_{i+1}\right)-Q\right]$,

$D=\frac{e_{i+1}^{\prime}\left(S_{i+1}-S_{i} e_{i}^{\prime}\right) A+S_{i} e_{i}^{\prime}\left(1-e_{i+1}^{\prime}\right) B}{S_{i} e_{i}^{\prime}\left(e_{i+1}^{\prime}-1\right)}$,

$\gamma_{1}=\left\{r_{i} S_{i}^{2}\left(r_{i+1}+r_{i}+p_{i+1}\right)+r_{i+1} S_{i+1}^{2}\left(r_{i}+p_{i}+r_{i+1}\right)-S_{i+1} S_{i}\left[\left(r_{i}+r_{i+1}\right)^{2}\right.\right.$

$\left.\left.+\left(r_{i}+r_{i+1}\right)\left(p_{i}+p_{i+1}\right)+\left(r_{i} p_{i+1}+r_{i+1} p_{i}\right)\right]\right\} \frac{1}{2 S_{i} S_{i+1}\left(r_{i+1}+r_{i}\right)\left(S_{i}-S_{i+1}\right)}$

$\gamma_{2}=\frac{\left(S_{i} r_{i}+S_{i+1} r_{i+1}\right) Q}{2 S_{i} S_{i+1}\left(r_{i+1}+r_{i}\right)\left(S_{i+1}-S_{i}\right)}$,

$Q=\sqrt{\left[S_{i}\left(r_{i+1}+r_{i}+p_{i+1}\right)-S_{i+1}\left(r_{i}+p_{i}+r_{i+1}\right)\right]^{2}+4 S_{i} S_{i+1} p_{i} p_{i+1}}$.

2) If $S_{i+1}<S_{i}$

$$
\begin{aligned}
\rho_{i}^{e}= & \frac{S_{i} e_{i} A e^{\gamma_{1} C_{i}}+S_{i+1} e_{i+1} B e^{\gamma_{2} C_{i}}+S_{i+1} e_{i+1} D e^{-\gamma_{2} C_{i}}}{A e^{\gamma_{1} C_{i}}+B e^{\gamma_{2} C_{i}}+D e^{-\gamma_{2} C_{i}}}, \\
p_{i}^{e}= & \left\{S _ { i } r _ { i } \rho _ { i } ^ { e } ( r _ { i } + p _ { i } ) ^ { 2 } ( r _ { i + 1 } + p _ { i + 1 } ) \left[p_{i} S_{i}^{2}+r_{i} S_{i} S_{i+1}+\rho_{i}^{e}\left(r_{i}+p_{i}\right)\left(S_{i}-S_{i+1}\right)\right.\right. \\
& \left.\left.-\rho_{i}^{e}\left(r_{i}+p_{i}\right) S_{i}\right]^{2}\right\} /\left\{\left[p_{i} S_{i}^{2}+r_{i} S_{i} S_{i+1}+\rho_{i}^{e}\left(r_{i}+p_{i}\right)\left(S_{i}-S_{i+1}\right)\right]\right. \\
& {\left.\left[S_{i}^{2} p_{i} r_{i} \rho_{i}^{e}\left(r_{i+1}+p_{i+1}\right)+S_{i+1} r_{i+1} p_{i+1}\left(r_{i}+p_{i}\right)^{2}\left(S_{i} r_{i}-\rho_{i}^{e}\left(r_{i}+p_{i}\right)\right)\right]\right\}, }
\end{aligned}
$$

$$
\begin{aligned}
r_{i}^{e}= & \left\{S _ { i } r _ { i } [ \rho _ { i } ^ { e } ] ^ { 2 } ( r _ { i } + p _ { i } ) ^ { 2 } ( r _ { i + 1 } + p _ { i + 1 } ) \left[p_{i} S_{i}^{2}+r_{i} S_{i} S_{i+1}\right.\right. \\
& \left.\left.+\rho_{i}^{e}\left(r_{i}+p_{i}\right)\left(S_{i}-S_{i+1}\right)-\rho_{i}^{e}\left(r_{i}+p_{i}\right) S_{i}\right]\right\} /\left\{\left[p_{i} S_{i}^{2}\right.\right. \\
& \left.+r_{i} S_{i} S_{i+1}+\rho_{i}^{e}\left(r_{i}+p_{i}\right)\left(S_{i}-S_{i+1}\right)\right]\left[S_{i}^{2} p_{i} r_{i} \rho_{i}^{e}\left(r_{i+1}+p_{i+1}\right)\right. \\
& \left.\left.+S_{i+1} r_{i+1} p_{i+1}\left(r_{i}+p_{i}\right)^{2}\left(S_{i} r_{i}-\rho_{i}^{e}\left(r_{i}+p_{i}\right)\right)\right]\right\}
\end{aligned}
$$

Then, the throughput of lines can be obtained by the new aggregation technique.

\section{NUMERICAL RESUlTS}

For the convenience of specifying the new technique, the data of the literature [6] are utilized in this paper. Meanwhile, both homogeneous model and non-homogeneous model of production lines are examined. Homogeneous lines mean that 
the processing rates of machines are all equal. Non-homogeneous lines then means the processing rates of machines are different.

We first present some typical results obtained in the case of homogeneous lines. Two lines $L_{1}$ and $L_{2}$, in which all the processing times $T$ are equal to 1 are examined firstly. Line $L_{1}$ has $N=4$ machines. Line $L_{2}$ has $N=10$ machines. The data and the numerical results for the lines are given in Tables I, II. Our method and the traditional method are evaluated by comparing their numerical results with the simulation respectively.

TABEL I: PRODUCTION LiNE $L_{1}(\boldsymbol{T}=\mathbf{1})$

\begin{tabular}{ccccc}
\hline \hline & 1 & 2 & 3 & 4 \\
\hline$p_{i}$ & 0.04 & 0.02 & 0.03 & 0.02 \\
$r_{i}$ & 0.08 & 0.04 & 0.06 & 0.04 \\
$C_{i}$ & 20 & 0 & 20 & \\
\hline \multicolumn{5}{c}{ Simulation } \\
Traditional Technique & 0.431 & \\
New Technique & & 0.327 & \\
\hline \hline
\end{tabular}

TABLE II: PRODUCTION LINE $L_{2}(\boldsymbol{T}=\mathbf{1})$

\begin{tabular}{ccccccccccc}
\hline \hline & 1 & 2 & 3 & 4 & 5 & 6 & 7 & 8 & 9 & 10 \\
\hline $1 / p_{i}$ & 900 & 50 & 200 & 3000 & 40 & 100 & 100 & 150 & 150 & 200 \\
$1 / r_{i}$ & 100 & 10 & 20 & 50 & 5 & 20 & 20 & 30 & 30 & 49 \\
$C_{i}$ & 90 & 20 & 0 & 0 & 20 & 100 & 130 & 150 & 600 & \\
\hline \multicolumn{1}{c}{ Simulation } & \multicolumn{1}{c}{0.698} \\
Traditional Technique & New Technique & 0.618 & & \\
\hline \hline
\end{tabular}

For the line $L_{1}$, the breakdown rates of the machines are large and the buffer capacity is much unbalanced. Compared with traditional technique, the result obtained by the new technique is closer to the simulation result. Line $L_{2}$ has smaller breakdown rates of machines compared with line $L_{1}$. Meanwhile, the ratio of the average production time $1 / p_{i}$ to the average breakdown time $1 / r_{i}$ is larger for each machine. It can be seen in the Table II that the traditional technique gets a better result. These examples mentioned above are only a few among those we tested. It can be drawn that for the lines with more or larger breakdown rates of machines whereas the repair rates of machines are comparatively fixed, the technique proposed by the paper provides better estimates of the throughput of line, e.g. Line $L_{1}$, than the traditional technique. The main reason is that larger breakdown rates or larger ratio of breakdown rate to repair rate of machines result in larger probability of blockage and starvation under the condition that the ratio of breakdown rate to repair rate of each machine are equal or nearly equal for homogeneous lines.

In the traditional aggregation method, the aggregation process is one-way aggregation and it stops when the whole production line is aggregated into a pseudo "equivalent" machine. However, the aggregation technique offered by the paper builds a new pseudo-line by "equivalent" machines obtained in former aggregation process. So the parameters of all machines are renewed repeatedly. That is to say, by our technique, all information of lines including blockage and starvation can be introduced to the new pseudo-line by "equivalent" machines obtained by former forward or backward aggregations. Thus the system process information of lines can be renewed rapidly and it to some extent reduces the influence incurred by the impact of blockage and starvation in aggregation process. So for production lines with large breakdown rates of machines, our aggregation technique gets a better estimation. But if the breakdown rates of machines are comparatively small, our aggregation technique has larger errors than the traditional technique.

We now consider the case of non-homogeneous lines. Several examples referred to as lines $L_{3}$ to $L_{4}$ are considered. These examples are again taken in part from [6]. Line $L_{3}$ has $N=4$ machines. Line $L_{4}$ has $N=6$ machines. The data and the numerical results for the lines are given in Table III and Table IV. Note that these examples are only a few among those we tested.

TABLE III: PRODUCTION LINE $\boldsymbol{L}_{3}$

\begin{tabular}{|c|c|c|c|c|c|c|}
\hline & \multicolumn{2}{|c|}{1} & 2 & \multicolumn{2}{|c|}{3} & 4 \\
\hline$p_{i}$ & \multicolumn{2}{|c|}{0.04} & 0.02 & \multicolumn{2}{|c|}{0.03} & 0.02 \\
\hline$r_{i}$ & \multicolumn{2}{|c|}{0.08} & 0.04 & \multicolumn{2}{|c|}{0.06} & 0.04 \\
\hline$C_{i}$ & \multicolumn{2}{|c|}{20} & 0 & \multicolumn{2}{|c|}{20} & \\
\hline$T_{i}$ & \multicolumn{2}{|c|}{1.0} & 0.95 & \multicolumn{2}{|c|}{1.05} & 1.0 \\
\hline \multicolumn{3}{|c|}{ Simulation } & & \multicolumn{2}{|r|}{0.426} & \\
\hline \multicolumn{3}{|c|}{ Traditional Technique } & & \multicolumn{2}{|r|}{0.317} & \\
\hline \multicolumn{3}{|c|}{ New Technique } & & \multicolumn{2}{|r|}{0.358} & \\
\hline \multicolumn{7}{|c|}{ TABLE IV: PRODUCTION LINE $\boldsymbol{L}_{4}$} \\
\hline & 1 & 2 & 3 & 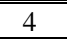 & 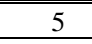 & 6 \\
\hline $1 / p_{i}$ & 9.24 & 45 & 45 & 45 & 45 & 9.24 \\
\hline $1 / r_{i}$ & 0.76 & 5 & 5 & 5 & 5 & 0.76 \\
\hline$C_{i}$ & 4 & 2 & 2 & 2 & 4 & \\
\hline$T_{i}$ & 0.356 & 0.28 & 0.28 & 0.28 & 0.28 & 0.347 \\
\hline \multicolumn{3}{|c|}{ Simulation } & & \multicolumn{2}{|r|}{2.116} & \\
\hline \multicolumn{3}{|c|}{ Traditional Technique } & & \multicolumn{2}{|r|}{1.68} & \\
\hline \multicolumn{3}{|c|}{ New Technique } & & \multicolumn{2}{|r|}{2.028} & \\
\hline
\end{tabular}

For the line $L_{3}$, there is a little difference on production rates of machines compared with Line $L_{1}$. Compared with the traditional method, our method also gets a better estimation for $L_{3}$ because probability of blockage and starvation actually increases due to the little variation of production rates. Line $L_{4}$ is a symmetrical line and the probability of blockage and starvation of the line is larger than that of Line $L_{3}$ because the buffers of $L_{4}$ on the whole have smaller capacity to afford the production capacity of machines than the buffers of $L_{3}$. It can be seen in Table IV that our method get a better estimation compared with the traditional method. The main reason is as same as that when analyzing homogeneous lines above.

In summary, our technique can get a fairly good estimation of throughput if the breakdown rates of machines are comparatively large when the ratios of breakdown rate to repair rate of machines are equal or nearly equal for homogeneous lines. For non-homogeneous lines, if the non-homogeneousness of lines results in high occurrence probabilities of blockage and starvation, then our technique can get a fairly good estimation. This is because the system 
information of lines can be renewed rapidly by our method and it consequently to some extent reduces the influence incurred by the impact of blockage and starvation in aggregation process, and deal with the lines well. Conversely, the traditional method gets better estimations for lines with low probability of blockage and starvation.

\section{CONCLUSIONS}

A new aggregation technique has been proposed to analyze the performance of hybrid production lines. The comparison analysis between the technique and the traditional aggregation method was done by numerical experiments. It can be drawn that the new technique has advantages to analyzing the lines with high non-homogeneousness or high occurrence probability of blockage and starvation. The new technique complements and develops the system analysis techniques of hybrid production lines.

\section{REFERENCES}

[1] G. Labinaz, M. M. Bayoumi, and K. Rudie, "A survey of modeling and control of hybrid systems," Annual Reviews in Control, vol. 21, pp. 79-92, 1997.

[2] Y. Dallery and S. B. Gershwin, "Manufacturing flow line systems: a review of models and analytical results," Queueing System, vol. 12, pp. 3-94, 1992.

[3] J. Li, D. E. Blumenfeld, N. Huang, and J. M. Alden, "Throughput analysis of production systems: recent advances and future topics," International Journal of Production Research, vol. 47, no. 14, pp. 3823-3851, 2009.

[4] T. Altiok, Performance analysis of manufacturing systems, New York: Springer, 1996.

[5] J. Li and S. M. Meerkov, Production System Engineering, New York: Springer, 2008.

[6] Y. Dallery, R. David, and X. L. Xie, "Approximate analysis of transfer lines with unreliable machines and finite buffers," IEEE Transactions on Automatic Control, vol. 34, no. 9, pp. 943-953, 1989.

[7] S. B. Gershwin, "An efficient decomposition method for the approximate evaluation of tandem queues with finite storage space and blocking," Operations Research, vol. 35, no. 2, pp. 291-305, 1987.

[8] B. Ancelin and A. Semery, "Calcul de la productivite d'une ligne integree de fabrication: CALIF, une méthode analytique industrielle ," RAIRO APPII, vol. 21, no. 3, pp. 209-238, 1987.

[9] Y. Dallery and H. L. Bihan, "An improved decomposition method for the analysis of production line with unreliable machines and finite buffers," International Journal of Production Research, vol. 37, no. 5, pp. 1093-1117, 1999.

[10] M. Colledani and T. Tolio, "A decomposition method to support the configuration/reconfiguration of production systems," CIRP Annals, Manufacturing Technology, vol. 54, no. 1, pp. 441-444, 2005.

[11] M. H. Burman, "New results in flow line analysis," Thesis (PhD), OR Center, MIT, 1995.
[12] H. B. Le and Y. Dallery, "A robust decomposition method for the analysis of production lines with unreliable machines and finite buffers," Annals of Operations Research, vol. 93, pp. 265-297, 2000

[13] J. Li, "Modeling and analysis of manufacturing systems with parallel lines," IEEE Transaction on Automatic Control, vol. 49, pp. 1824-1829, 2004

[14] S. B. Gershwin and M. H. Burman, "A decomposition method for analyzing inhomogeneous assembly/disassembly system," Annals of Operation Research, vol. 93, pp. 91-115, 2000.

[15] J. Li, "Performance analysis of production systems with rework loops," IIE Transaction, vol. 36, pp. 755-765, 2004.

[16] J. Li and S. M. Meerkov, "Due-time performance in production systems with Markovian machines," in Analysis and Modeling of Manufacturing Systems, S. B. Gershwin, Y. Dallery, C. T. Papadopolos and J. M. Smith, Eds. Dordrecht: Kluwer Academic, 2003, pp. 221-253.

[17] J. Liu, Z. Y. Rui, J. T. Zhao, "System Aggregation method for failure prone production lines with unreliable limited buffers," Chinese Journal of Mechanical Engineering, vol. 21 , no. 2, pp. 81-86, 2008

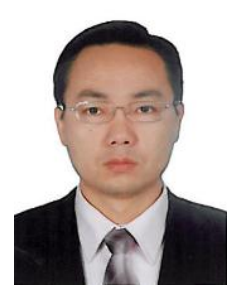

Jun Liu is currently a professor in Lanzhou University of Technology, Lanzhou, China. He received his Ph.D. degree in engineering from Zhejiang University, Hangzhou, China, in 2005. His current research interests include complex manufacturing system, production scheduling and control, lean production, etc.

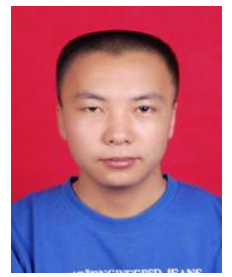

Qingying Fan received his bachelor degree from Lanzhou University of Technology, Lanzhou, China, in 2012. He is currently a graduate student in Lanzhou University of Technology. His current research interests include complex manufacturing system, hybrid system, etc.

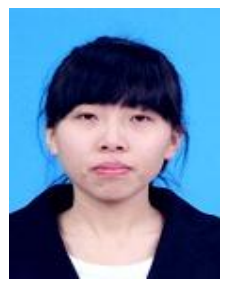

Junping Kong received her bachelor degree from Lanzhou University of Technology, Lanzhou, China, in 2012. She is currently a graduate student. Her current research interests include complex manufacturing system, production scheduling and control, etc. 